\title{
Factores asociados a la presentación de cojeras en 50 rebaños lecheros de la X Región, Chile ${ }^{\#}$
}

\author{
Factors associated to lameness in 50 dairy herds in the $\mathrm{X}^{\text {th }}$ Region, Chile

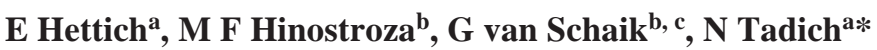 \\ anstituto de Ciencias Clínicas Veterinarias, Facultad de Ciencias Veterinarias, Universidad Austral de Chile. \\ ${ }^{b}$ Instituto de Medicina Preventiva Veterinaria, Facultad de Ciencias Veterinarias, Universidad Austral de Chile. \\ ${ }^{c}$ Actualmente: Animal Health Service Ltd., Deventer, The Netherlands.
}

\begin{abstract}
SUMMARY
The aim of this study was to determine the risk factors related to the prevalence of foot lesions in 50 dairy farms in three provinces (Valdivia, Osorno and Llanquihue) of the $\mathrm{X}^{\text {th }}$ Region, Chile. Each farm was visited once between April and July, 2004. During each visit, all lactating cows were observed during locomotion. In those cows showing a degree of lameness, all four feet were examined. The degree of lameness was scored in scale of 1 (slightly lame) to 4 (very lame). Data were recorded in an individual recording sheet for each cow. All the facilities and paddocks where the cows were kept were inspected during each visit. A questionnaire was handed to the manager or the owner of the farm. All the data recorded and the answers to the questionnaire were introduced in an Excel spread sheet as numerical variables and analyzed using the statistical program SPSS 8.0. Risk factors for the four more prevalent causes of lameness were determined using a logistic regression model. The associated factors for an increase of the prevalence in the dairy herds were determined with a multivariable linear regression model. A total of 7501 dairy cows were observed, with 641 of them being diagnosed with some degree of lameness. Cows with three or more lactations were 3.8 times more likely to develop chronic lesions of the hooves and cows with two lactations were 2 times more likely to develop overgrowth of the sole, compared with other lactations. German Red-pied cows were 1.7 times more likely to have white line lesions and 2 times more wall lesions than other breeds. Three risk factors related to lameness were identified: i) changing the diet 25 days prior to calving vs changing the diet nearer to calving date or not changing the diet, ii) concrete roads vs gravel and mud roads, iii) the absence of a footbath versus the presence of a footbath.
\end{abstract}

Palabras clave: cojeras, rebaño lechero, factores de riesgo.

Key words: lameness, dairy cattle, risk factors.

\section{INTRODUCCION}

La población bovina de Chile es de 4.098 .438 cabezas, de las cuales $380.000(61,5 \%)$ son vacas lecheras que están ubicadas en la Décima Región, produciendo aproximadamente el $64 \%$ de la leche que abastece a la industria nacional (INE 1997, UACh 1999).

La intensificación en la producción lechera ha determinado un incremento en la incidencia de cojeras, con un considerable impacto económico y en el bienestar animal (Pyman 1997, Vermunt 1999, Galindo y Broom 2000). Las patologías podales en las vacas lecheras han sido consideradas como la tercera causa de pérdidas económicas en el ganado lechero, después de las mastitis y la infertilidad, siendo el mayor problema en cuanto a bienestar animal (Vermunt 1992, Kossaibati y Esslemont 2000, O' Callaghan 2002, Whay y col 2003).

\footnotetext{
Aceptado: 28.11.2006.

\# Financiado por Proyecto FONDECYT 1040176

* Casilla 567, Valdivia, Chile; ntadich@uach.cl
}

Las pérdidas económicas producidas por las cojeras están determinadas por la menor producción de leche (Tranter y Morris 1991, Rajala-Schultz y col 1999, Warnick y col 2001, Green y col 2002), menor rendimiento reproductivo (Hassall y col 1993, Sprecher y col 1997), mal aprovechamiento del alimento o menor consumo de éste, producto de la deambulación deteriorada, eliminación prematura del animal del rebaño, susceptibilidad a otras enfermedades y mayor cantidad de leche rechazada por la planta, producto de la presencia de residuos de antibióticos utilizados en los tratamientos (Esslemont 1990, Greenough y col 1997, Vermunt y Parkinson 2002).

Las cojeras del bovino son afecciones multifactoriales, donde las prácticas de alimentación, el medio ambiente, los procesos infecciosos, la genética y el comportamiento tanto animal como humano representan factores de riesgo desencadenantes (Acuña 2002). Harris y col (1988) señalan que las cojeras en vacas lecheras se pueden deber a traumas, infecciones, deficiencias nutricionales, desórdenes metabólicos y que la incidencia en el rebaño puede variar con la edad y la raza de las vacas, el terreno, condiciones climáticas y el manejo del predio. Vermunt 
(1992) señala, además, que otros factores tales como la estación del año, la etapa de la lactancia, y la conformación de la pezuña contribuyen a la incidencia de cojeras.

Entre los factores de riesgo de origen nutricional se ha mencionado que altos niveles de energía y bajos de fibra en la ración causan acidosis ruminal, produciendo laminitis y la subsecuente aparición de cojeras debido a úlceras plantares y abscesos de la línea blanca (Vermunt 1990, Blowey 1998). Según Manson y Leaver (1988), niveles altos de proteínas en la ración jugarían un papel en el desarrollo de las cojeras. Sin embargo, Bazeley y Pinsent (1984) y Logue y col (1989) indican que este papel no sería muy importante. Otras causas de tipo nutricional son producidas por deficiencias de minerales, tales como el zinc y el cobre, los cuales, junto a la metionina, son responsables de la queratinización de los tejidos (Greenough 1997).

Entre los factores de manejo, el confinamiento de las vacas por largos períodos de tiempo aumenta la presentación de problemas podales (Nelson y Petersen 1984, Blowey 1998). El despalme periódico es probablemente la medida más importante para prevenir la aparición de laminitis y afecciones secundarias de las pezuñas (Bergsten y Frank 1996, Tadich 2003). En países donde se realiza pastoreo durante todo el año, las laminitis en vacas de lechería están asociadas al largo, la calidad y el diseño de los caminos (Leonard y col 1994).

La incidencia de cojeras es más alta en invierno cuando las vacas se encuentran estabuladas; sin embargo, el período de verano también es de importancia. Factores que se encuentran asociados a este período son los caminos, el personal a cargo de los animales, y las grandes distancias que tienen que caminar las vacas (Clarkson y Ward 1991).

Cierta proporción de vacas parece mostrar predisposición genética a determinadas lesiones, como úlcera plantar y laminitis (Baggott 1982, Brochart 1987, Vermunt y Greenough 1994). El efecto genético en lesiones podales ha sido estudiado en vacas lecheras, y se concluyó que la incidencia de lesiones de la suela era toro-dependiente, pero que también había una interacción entre el toro y el sistema de estabulación (Leonard y col 1994).

El comportamiento ha sido sugerido como un factor predisponente a cojeras. Los efectos de confrontación entre vaquillas sumisas y vacas dominantes del rebaño, podría ser considerado como un carácter heredable (Greenough 1997). David (1986) señala que la introducción a un tipo de estabulación con cubículos desconocidos para el animal puede que no sea bien aceptado en las primeras semanas, lo cual llevaría a las vacas a estar más tiempo de pie sobre las superficies de concreto. Un inadecuado número de cubículos y pasillos muy angostos pueden crear un problema en vacas de primer parto; para evitar la confrontación con vacas dominantes, ellas rechazarían el uso de cubículos. El diseño de los cubículos es muy importante en la presentación de cojeras en las vacas estabuladas (Baggott 1982, Blowey 1998, Tadich 2003). Además, las vacas de primer parto son a menudo las últimas en entrar a la sala de ordeña, por lo tanto están por períodos prolongados paradas sobre superficies de concreto (Vermunt, 1990).

Overton y col (2002) observaron que el estrés calórico modifica el comportamiento de las vacas, lo que aumenta el riesgo de laminitis y lesiones de la pezuña. Schultz (1984) ha demostrado que el porcentaje de vacas que está de pie aumenta con el incremento de la temperatura ambiental. Por otra parte, Nilsson (1963) asoció la laminitis al momento del parto con la presentación de metritis y retención de membranas fetales.

El estado de la lactancia es otro factor importante, las cojeras se presentan generalmente entre dos a 16 semanas después del parto (Dewes 1978, Offer y col 2001, Vermunt y Parkinson 2002). Es difícil de separar la influencia de factores que confluyen, como la estabulación, parto, etapa de lactancia y nivel de producción en la ocurrencia de laminitis.

En Chile existen escasos estudios publicados sobre los factores de riesgo asociados a la presentación de patologías podales (Rodríguez y col 1999, Berendsen y col 2005). El objetivo de este estudio fue proveer información preliminar acerca de aquellos factores de riesgo asociados a la presentación de las patologías podales más frecuentes en rebaños lecheros, así como identificar a los factores de riesgo asociados a una alta prevalencia de cojeras en estos rebaños.

\section{MATERIAL Y METODOS}

Se utilizaron 50 lecherías de la Décima Región de Chile, distribuidas de la siguiente forma: 17 en la provincia de Valdivia, 17 en la provincia de Osorno y 16 en la provincia de Llanquihue. Las lecherías que participaron en este estudio fueron escogidas por conveniencia, y el número de vacas existentes en ellas fluctuó entre 70 y 380 vacas masa. Las lecherías fueron visitadas por una sola vez durante el estudio, de acuerdo a un programa de visitas establecido de común acuerdo con el propietario o encargado del predio y el veterinario asesor.

Durante la visita se examinaron mediante inspección todas las vacas en ordeña, para determinar la presencia de algún tipo de claudicación en alguno de sus miembros. El grado de claudicación se clasificó de acuerdo al grado de apoyo de dicho miembro al desplazamiento y a la estación de acuerdo a la pauta descrita por Sprecher y col (1997) y modificada por Tadich y col (2005) donde el grado 0 correspondió a una vaca sana y el grado 4 a una vaca extremadamente coja. Las vacas que presentaban algún grado de claudicación fueron separadas del rebaño e introducidas al brete de examen de pezuñas, cuando éste existía en el predio, y sometidas a un exa- 
men clínico de cada uno de sus miembros para diagnosticar el tipo de lesión presente.

Los hallazgos fueron registrados en una ficha individual en la que se incluían los datos del propietario, del predio, la fecha de visita, el número del arete de la vaca examinada, el grado de claudicación, el miembro y pezuña afectados, el tipo de lesión encontrada y la ubicación de la lesión. Durante la visita se realizó una inspección de todas las construcciones, instalaciones y potreros en los cuales eran manejadas las vacas en lactancia, al mismo tiempo se realizó una encuesta, previamente validada, al encargado del predio o al propietario del mismo. En esta encuesta se registraron datos relacionados con la ubicación del predio, número de ordeñas diarias, distancia promedio $(\mathrm{m})$ que caminaban las vacas diariamente, promedio de producción de leche diario en el predio, sistema de cubiertas, raza predominante de las vacas, número de vacas en lactancia, número de vacas en el primer tercio de la lactancia, número de vacas cojas, tipo de estabulación, tipo de cama, medida de las camas, frecuencia de limpieza de aquellas áreas en que se manejaban las vacas de lechería, tipo de piso de esas áreas, tipo de caminos y/o callejones existentes en el predio, tipo de suelo de los potreros, dieta que recibían tanto las vacas en lactancia como aquellas que se encontraban en el período preparto, fecha de cambio de dietas en el período preparto, edad y/o grupo de animales que presentaban más cojeras, frecuencia de arreglo de patas, qué persona(s) realizaba(n) el arreglo, capacitación de la(s) persona(s) que lo hacían, presencia de brete en el predio, presencia de pediluvio en el predio, frecuencia de uso del pediluvio y productos usados en el pediluvio.

\section{ANALISIS ESTADISTICO}

Las respuestas obtenidas con la encuesta fueron ingresadas como variables numéricas a una planilla de Microsoft ${ }^{\circledR}$ Excel XP, para su posterior análisis. La prevalencia predial de vacas cojas, así como la prevalencia total de los predios, fue la informada por Tadich y col (2005).

Para la determinación de los factores de riesgo dependientes de la vaca, para las cuatro patologías más prevalentes (deformación crónica de la pezuña, lesión de la línea blanca, lesión de muralla, doble suela), se utilizó un modelo de regresión logística, siendo la variable dependiente una de las cuatro patologías más frecuentes (presencia o ausencia) y las variables independientes, la ubicación geográfica (provincia) de los predios, la raza del animal y el número y etapa de lactancia. Los factores de riesgo significativamente asociados fueron obtenidos mediante un análisis de $\chi^{2}$ entre la causa de la cojera y la presencia o no del factor de riesgo. Aquellos factores con valores $\mathrm{P} \leq 0,20$ fueron seleccionados para posteriormente ingresarlos al modelo de regresión logística, los cuales fueron excluyéndose hasta lograr un nivel de significancia de $\mathrm{P} \leq 0,05$. Para la determinación de los factores de riesgo dependientes del predio se llevó a cabo una regresión lineal simple, usando las prevalencias prediales como variable dependiente y los factores de riesgo como variables independientes. Aquellas variables cuyas regresiones tuvieron un valor $\mathrm{P} \leq 0,20$ fueron seleccionadas para posteriormente ingresarlas a un modelo de regresión lineal multivariable, las cuales fueron excluyéndose hasta llegar a un modelo final que tuviera todas las variables con un nivel de significancia de $\mathrm{P}<0,05$. Para el análisis estadístico de los datos se utilizó el programa estadístico SPSS 8.0. La bondad del modelo con los datos fue determinado con la varianza explicada por el modelo final ( $\mathrm{R}^{2}$ ajustado) y plots de los residuales y las predicciones del modelo. Además, para chequear el presupuesto de la normalidad de la variable dependiente (prevalencia), la normalidad de las residuales fue determinada con una prueba de Shapiro-Wilk.

\section{RESULTADOS Y DISCUSION}

De acuerdo a lo observado en el cuadro 1, la raza y el número de lactancias fueron los factores de riesgo más importantes en la presencia de las cuatro patologías con más alta prevalencia en la Décima Región.

La patología más frecuente fue la deformación crónica de la pezuña (19,8\%), la que engloba a aquellas alteraciones, como la pezuña en tijera, pezuña en zapatilla, pezuña en tirabuzón, pezuña larga y laminitis. El número de lactancias y por ende la edad fue un factor de riesgo para la presentación de esta patología, donde las

Cuadro 1. Modelo final para los factores de riesgo significativamente asociados $(\mathrm{P} \leq 0,05)$ a las cuatro patologías de mayor prevalencia diagnosticadas en 7501 vacas lecheras de la Décima Región de Chile.

Final model for risk factors significantly associated $(\mathrm{P} \leq 0.05)$ with the four most prevalent lesions diagnosed in 7501 dairy cows of the $\mathrm{X}^{\text {th }}$ Region, Chile.

\begin{tabular}{|c|c|c|c|c|c|c|}
\hline Patología & Factor de Riesgo & Variable & $\beta$ & E. E. & $\mathrm{P}$ & O R \\
\hline Deformación crónica & Número de lactancias & Tres o más & 1,34 & 0,42 & 0,00 & 3.83 \\
\hline Lesión de línea blanca & Raza & Overo Colorado & 0,55 & 0,27 & 0,05 & 1,71 \\
\hline Lesión de muralla & Número de lactancias & Segunda & 0,78 & 0,39 & 0,04 & 2,19 \\
\hline Doble suela & Raza & Overo Colorado & 0,71 & 0,31 & 0,02 & 2,05 \\
\hline
\end{tabular}


vacas con tres o más lactancias presentaron 3,8 veces más posibilidades de padecer esta enfermedad. Esto concuerda con Sogstad y col (2005), quienes encontraron que las vacas con tres o más lactancias tuvieron mayores riesgos de cojeras que aquellas con menos de tres lactancias. Por otra parte, Van Amstel y Shearer (2001) señalan que estas patologías tienen una causa multifactorial, pero las condiciones de estabulación juegan un rol fundamental. También la superficie en donde se desplaza la vaca tiene un efecto en la calidad de la pezuña, lo que está determinado por las condiciones de estabulación (Petersen y Nelson 1984).

La segunda alteración en importancia fue la lesión de la línea blanca $(15,9 \%)$. La raza fue un factor significativo en la presentación de esta patología; la raza Overo Colorado presentó 1,7 veces más posibilidades que otras razas para la presentación de la lesión de la línea blanca. Esto explica la alta prevalencia de esta patología en la provincia de Llanquihue en comparación con las otras provincias estudiadas, ya que en Llanquihue se presentó la mayor cantidad de vacas de la raza Overo Colorado. Probablemente influye en este hallazgo el escaso manejo de las patas que se efectúa a las vacas en la provincia de Llanquihue. Sin embargo, habría que señalar que si bien la literatura no describe predisposición racial para esta patología específica, sí lo hace para laminitis y cojeras en general (Enevoldsen y col 1991, Gitau y col 1996, Greenough 1997, Ossent y col 1997). Berendsen y col (2005) encontraron que la enfermedad de la línea blanca estaba asociada a la segunda etapa de la lactancia.

La lesión de muralla constituyó la tercera patología con más alta prevalencia $(10,9 \%)$. Las vacas en su segunda lactancia tuvieron dos veces más posibilidades de desarrollar esta patología que las vacas con otros números de lactancias. Esto podría deberse a que esta patolo- gía se presenta entre otras cosas por desórdenes nutricionales (laminitis, deficiencias de minerales, etc.), los que podrían producirse en la primera lactancia de la vaca y prolongarse hasta la siguiente lactancia (Greenough 2001). En este estudio se observó que en la mayoría de los casos de lesión de muralla había participación de algún elemento extraño en la lesión, y lo más comúnmente encontrado eran piedras, por lo que los caminos y las distancias que deben recorrer las vacas desde y hacia la sala de ordeño podrían jugar un papel importante en el desarrollo de la patología. Según Greenough (2001), esta enfermedad puede ser provocada por enfermedades sistémicas, estrés o desórdenes nutricionales, lo que podría explicar la mayor presentación de esta patología en animales jóvenes, ya que éstos son sometidos a un cambio de alimentación al entrar a la vida productiva.

La patología denominada doble suela constituyó la cuarta patología con más alta prevalencia (10\%). La raza Overo Colorado presentó dos veces más posibilidades de sufrir esta patología que las otras razas presentes en el estudio. En este caso probablemente sucede lo mismo que en el caso de la lesión de la línea blanca, en el sentido de que no es la genética, sino que el manejo deficiente a que son sometidas las vacas de la raza Overo Colorado lo que interviene en la presentación de la doble suela. Manske y col (2002) al comparar vacas a las cuales se les realizó arreglo de pezuñas durante el otoño vs aquellas en las que no se les realizó, encontraron que estas últimas presentaron mayor riesgo de sufrir de cojeras en general, hemorragias de la suela, úlceras plantares y enfermedad de la línea blanca, esto reafirma la importancia del despalme preventivo.

Como se observa en el cuadro 2 los factores de riesgo dependientes del predio, asociados a altas prevalencias

Cuadro 2. Modelo final de la regresión lineal multivariable de los factores asociados a la prevalencia de cojeras en 50 rebaños lecheros de la Décima Región de Chile.

Final multivariable linear regression model for those factors significantly $(\mathrm{P} \leq 0.05)$ associated with the prevalence of lameness in 50 dairy herds of the $\mathrm{X}^{\text {th }}$ Region, Chile.

\begin{tabular}{|c|c|c|c|c|c|}
\hline & \multicolumn{2}{|c|}{ Coeficientes no estandarizados } & \multirow{2}{*}{$\mathrm{t}$} & \multirow{2}{*}{ Sig. } & \multirow{2}{*}{$\mathrm{R} \leq$ ajustado $*$} \\
\hline & $\mathrm{B}$ & Error estándar & & & \\
\hline Constante & 9,78 & 2,59 & 3,773 & 0,00 & 0,38 \\
\hline $\begin{array}{l}\text { Cambio alimentación } 25 \text { días antes del part } \\
\text { cambio alimentación cercano al parto o no } \\
\text { se cambia la alimentación }\end{array}$ & 4,22 & 1,58 & 2,68 & 0,01 & \\
\hline $\begin{array}{l}\text { Caminos de concreto vs caminos de ripio, } \\
\text { tierra y/o maicillo }\end{array}$ & 5,93 & 2,30 & 2,58 & 0,01 & \\
\hline $\begin{array}{l}\text { No presencia de pediluvio en el predio vs } \\
\text { presencia de pediluvio }\end{array}$ & 3,48 & 1,63 & 2,13 & 0,04 & \\
\hline
\end{tabular}

* La varianza explicada por el modelo final. 
de patologías podales fueron, los cambios de alimentación 25 días antes del parto, la presencia de caminos de concreto y la no presencia de pediluvios. Se puede concluir que la asociación linear entre estas variables fue buena, ya que el $\mathrm{R}^{2}$ ajustado fue de un $38 \%$, lo que estaría explicando que un $38 \%$ de la variabilidad en la prevalencia entre los rebaños estaría dado por estos factores. Además, el resultado de la prueba Shapiro-Wilk mostró que la distribución de los residuales fue normal ( $\mathrm{W}=0,96, \mathrm{P}=0,10)$.

El cambio de alimentación 25 días antes del parto, a diferencia de los factores cambio de alimentación más cercano al parto o no se realiza cambio de alimentación, se asoció a un aumento de las cojeras en los rebaños lecheros. De acuerdo con Greenough (1997), las dietas de transición del preparto deben ser formuladas y entregadas a las vacas de forma tal que eviten la presentación de acidosis ruminal. La acidosis jugaría un papel importante en la presentación de laminitis en las vacas (Ossent y col 1997). Por este motivo resulta extraño que el uso de dietas de transición 25 días preparto haya resultado ser un factor de riesgo. Los factores de riesgo en este tipo de análisis pueden ser el resultado del azar, sesgos del análisis o asociaciones verdaderas, los cuales conducen a plantearse nuevas hipótesis. En este caso no podemos determinar a cuál de ellos se debe este resultado, por lo que, con los antecedentes disponibles, no podemos afirmar que la introducción de dietas de transición 25 días antes del parto sea la causa de un aumento de las cojeras en el posparto.

Cabe señalar que existe en la literatura consultada escasa información sobre el efecto de las dietas de transición sobre la presentación de cojeras en las vacas posparto. Donovan y col (2004) señalan que dietas con baja energía neta en el preparto y alta energía neta en el posparto inmediato podrían incrementar el riesgo de laminitis subclínicas si no son manejadas adecuadamente. Una razón para los resultados obtenidos podría estar dada por una inadecuada utilización de las dietas de transición durante el período preparto. El aumento repentino del consumo de carbohidratos, o bien el consumo continuado de altos niveles de los mismos, produce un cambio en la población microbiana del rumen y una acidosis consecuente (Greenough 1997), que puede instaurar una laminitis aguda. Esto ocurre especialmente pre y posparto. La acidosis también se produce cuando se utilizan granos altamente degradables, como la cebada y el maíz (Westwood y col 2003). Muchos problemas ocurren en el período de transición (4 semanas preparto a 4 semanas posparto), cuando la flora del rumen no está adaptada a una dieta rica en energía, por lo tanto, el plano nutricional debe ir cambiando gradualmente hacia la dieta de lactancia (Shearer 1998). Por otra parte, este factor de riesgo podría ser un indicador indirecto del grado de intensificación de la producción de leche del predio, ya que aquellos predios con mayor producción de leche, generalmente, tienen un manejo nutricional más intensivo con mayor utilización de concentrado. Flor (2006) encontró que el tamaño del rebaño estuvo asociado a mayores prevalencias de cojeras y que este factor de riesgo se asociaba positivamente a los cambios de alimentación 25 días antes del parto, entre otros.

La ausencia de pediluvios en el predio versus la presencia de éstos, estuvo asociada a un aumento en la prevalencia de cojeras. Los pediluvios bien utilizados constituyen una herramienta que contribuye en el tratamiento $\mathrm{y}$, sobre todo, en el control de las afecciones podales necrótico-corrosivas del bovino (Münzenmayer 1997). Contrario a lo observado en este estudio, Chesterton y col (1989) encontraron que hubo una asociación positiva entre la presencia de pediluvios y las cojeras, lo cual fue visto más como un efecto a las altas prevalencias de cojeras, que una causa. Esto se puede interpretar como que en aquellos predios que tienen problemas de cojeras es más común encontrar el uso de pediluvios. Nelson y Petersen (1984), Sumner y Davies (1984) cuestionan la efectividad de los pediluvios usados para el control general de las enfermedades de los pies del bovino, porque las recomendaciones son empíricas y no hay evidencia publicada que las soporte. Es importante destacar que sólo un $26 \%$ de los predios visitados contaba con la presencia de un pediluvio, lo que indica una baja utilización de este sistema como una medida preventiva y terapéutica para las afecciones podales. El valor del pediluvio sólo es importante en casos de cojeras de origen infeccioso (Radostits y col 2000), no así en aquellas cojeras producto de laminitis agudas o crónicas.

La presencia de caminos de concreto a diferencia de caminos de ripio, maicillo y/o tierra se asoció a un aumento de las prevalencias de cojeras. El concreto es una superficie abrasiva, y por lo tanto causa un rápido desgaste de la pezuña de los animales con el consecuente daño. Por lo tanto, los animales se encuentran más propensos a desarrollar problemas podales. Esto coincide con Bergsten (1988), el cual encontró una correlación positiva entre lesiones similares a laminitis y suelos de concreto. Sin embargo, hay que considerar la posibilidad de que en aquellos predios en que existían altas prevalencias de cojeras se haya optado por el uso de este tipo de caminos como una forma de revertir esa situación.

\section{RESUMEN}

Con el objeto de determinar los factores de riesgo asociados a la prevalencia de lesiones podales se llevó a cabo un estudio en 50 lecherías de tres provincias (Valdivia, Osorno y Llanquihue) de la $\mathrm{X}$ Región, Chile. Las lecherías fueron visitadas una sola vez entre abril y julio, 2004. En cada visita todas las vacas en ordeño fueron observadas durante su locomoción. En aquellas vacas que presentaban algún grado de cojera se examinaron todas las patas. El grado de cojera fue clasificado de 1 (levemente coja) a 4 (muy coja). Los datos fueron 
anotados en hojas de registro individual para cada vaca. En cada visita las construcciones y potreros donde se mantenían las vacas fueron inspeccionados y al mismo tiempo se llevó a cabo una encuesta al dueño o al encargado de la lechería. Todos los datos registrados y los resultados de la encuesta fueron introducidos en una planilla Excel como variables numéricas y analizadas utilizando el programa estadístico SPSS 8.0. Los factores de riesgo para las cuatro causas de cojeras más prevalentes fueron determinados usando un modelo de regresión logística. Los factores asociados a la prevalencia de los rebaños fueron determinados utilizando un modelo de regresión multivariable. Fueron observadas un total de 7.501 vacas, de las cuales $641(8,54 \%)$ fueron diagnosticadas con algún grado de cojera. Vacas con tres o más lactancias tuvieron 3,8 veces más lesiones crónicas de la pezuña y vacas con dos lactancias tuvieron 2 veces más sobrecrecimiento de la suela, comparadas con otras lactancias. Las vacas de la raza Overo Colorado tuvieron 1,7 más lesiones de la línea blanca y 2 veces más lesiones de la pared comparadas con vacas de otras razas. Se identificaron tres factores de riesgo para la prevalencia de cojeras en los rebaños estudiados, cambios de la dieta 25 días antes del parto vs cambios de la dieta cerca del parto o no cambio de la dieta antes del parto, caminos de concreto vs caminos de tierra o piedras, ausencia de pediluvios vs presencia de pediluvios.

\section{REFERENCIAS}

Acuña R. 2002. Estudio de rengueras en rodeos lecheros: una guía para el veterinario asesor. $X$ Congreso Latinoamericano de Buiatría y XXX Jornadas Uruguayas de Buiatría, Uruguay, Pp 44-53.

Baggott D. 1982. Hoof lameness in dairy cattle. In Practice 4, 133-140.

Bazeley K, P J N Pinzent. 1984. Preliminary observations on a series of outbreaks of acute laminitis in dairy cattle. Vet Rec 115 , 619-622.

Berendsen N, E Flor, J Borkert, G Van Schaik, N Tadich, S Bennema, L Dambacher. 2005. Fatores de risco asociados a algumas patologias podais de vacas leiteiras no sul do Chile. Resúmenes III Encuentro de Podólogos de Rumiantes del Mecosur, IV Congreso de Buiatría del Estado de Río de Janeiro. Sociedad Brasilera de Buiatría, Buzios, Brasil.

Bergsten C. 1988. Sole bruising as indicator of laminitis in cattle. A field study. In: Proceedings of the XVth World Buiatrics Congress. Palma de Mallorca, España, Pp 1072-1076.

Bergsten C, B Frank. 1996. Sole hemorrhages in tied primiparous cows as an indicator of periparturient laminitis: effects of diets, flooring and season. Acta Vet Scand 37, 383-394.

Blowey R W. 1998. Diseases of the digit: description of common lesions. In: Melling M, M Alder (eds). Bovine Practice 2. Saunders, England, Pp 51-68.

Brochart M. 1987. Foot lameness of the cow, a multifactorial disease. In: Wierenga H K, Boston D J (eds). Martinus Nijhoff Publishers, UK.

Chesterton R N, D U Pfeiffer, R S Morris, C M Tanner. 1989. Environmental and behavioural factors affecting the prevalence and foot lameness in New Zealand dairy herds a case control study. New Zeal Vet J 37, 135-142.

Clarkson M J, W R Ward. 1991. Farm tracks, stockman's herding and lameness in dairy cattle. Vet Rec 138, 563-567.

David G P. 1986. Cattle behaviour and lameness. In: Proceedings of the Vth International Symposium on Disorders of the Ruminant Digit. Dublin, Ireland, Pp 79-86.

Dewes H F. 1978. Some aspects of lameness in dairy herds. New Zeal Vet $J$ 26, 147-148.

Donovan G A, C A Risco, G M Temple, T Q Tran, H H van Horn. 2004. Influence of transition diets on ocurrence of subclinical laminitis in Holstein dairy cows. J Dairy Sci 87, 73-84.
Enevoldsen C, T Grohn, I Thysen. 1991. Heel erosion and other interdigital disorders in dairy cows: associations with season, cow characteristics, disease and production. J Dairy Sci 74, 1299-1309.

Esslemont R J. 1990 The costs of lameness in dairy herds. Proceedings of the VIth International Symposium on Diseases of the Ruminant Digit. Liverpool, UK, Pp 237-251.

Flor, E. 2006. Claudicaciones en vacas de rebaños lecheros de la Décima Región, Chile: Prevalencia, Lesiones y Factores de Riesgo. Tesis de Magíster en Ciencias. Universidad Austral de Chile.

Galindo F, D M Broom. 2000. The relationships between social behaviour of dairy cows and the occurrence of lameness in three herds. Res Vet Sci 69, 75-79.

Gitau T, J J McDermott, S M Mbiuki. 1996. Prevalence, incidence, and risk factors for lameness in dairy cattle in small-scale farms in Kikuyu Division, Kenya. Prev Vet Med 28, 101-115.

Green L E, V J Hedges, Y H Schukken, R W Blowey, A J Packington. 2002. The impact of clinical lameness on the milk yield of dairy cows. J Dairy Sci 85, 2250-2256.

Greenough P. 1997. Lameness in Cattle. In: Weaver D. $3^{\text {rd }}$ ed. Saunders Company, England.

Greenough P R. 2001. Sand cracks, horizontal fissures, and other conditions affecting the wall of the bovine claw. The Veterinary Clinics of North America Food Animal Practice 17, 93-110.

Greenough P R, A D Weaver, R M Broom, R J Esslemont, F Galindo. 1997. Basic concepts of bovine lameness. In: Lameness in cattle. Greenough P R, Weaver A D (eds). WB Saunders, Philadelphia, USA, Pp 3-13.

Harris D J, C D Hibburt, G A Anderson. 1988. The incidence, cost and factors associated with foot lameness in dairy cattle in south western Victoria. Aust Vet J 65, 171-176.

Hassal S A, W R Ward, R D Murray. 1993. Effects of lameness on the behaviour of cows during summer. Vet Rec 132, 578-580.

INE, Instituto Nacional de Estadísticas, Chile. 1997. VI Censo Nacional Agropecuario. Chile.

Kossaibati M A, R J Esslemont. 2000. The incidence of lameness in 50 dairy herds in England. Proceedings of the III International Conference on Bovine Lameness. Parma, Italy, Pp 160-162.

Leonard F C, J O'Connell, K O'Farrell. 1994. Effect of different housing conditions on behaviour and foot lesions in Friesian heifers. Vet Rec 134, 490-494.

Logue D N, A Lawson, D J Roberts, E A Hunter. 1989. The effect of two different protein sources in the diet upon incidence and prevalence of lameness in dairy cattle. Anim Prod 48, 636.

Manske T, J Hultgreen, C Bergsten. 2002. The effect of claw trimming on the hoof health of Swedish dairy cattle. Prev Vet Med 54, 113-129.

Manson F J, J D Leaver. 1988. The influence of dietary protein intake and of hoof trimming on lameness in dairy cattle. Anim Prod 47,191-199.

Münzenmayer W. 1997. Afecciones podales en rodeos lecheros: desafío profesional. Therios (suppl): 1-31.

Nelson D, G Petersen. 1984. Foot diseases in cattle. Part I. Examination and special procedures. The Compendium on Continuing Education, Food Animal Practice 6, 545-552.

Nilsson S A. 1963. Clinical, morphological and experimental studies of laminitis in cattle. Acta Vet Scand 4, 9-304.

O'Callaghan K. 2002. Lameness and associated pain in cattle challenging traditional perceptions. In Practice 24, 212-219.

Offer J E, E J Fisher, S A Kempson, D N Logue. 2001. The effect of feeding grass silage in early pregnancy on claw health during first lactation. Vet J 161, 186-193.

Ossent P, P Greenough, J Vermunt. 1997. Laminitis. In: Lameness in Cattle. $3^{\text {rd }}$ ed. Saunders Company. England. Pp 277-292.

Overton M W, W M Sischo, D A Moore. 2002. Using time lapse video photography to asses dairy cattle lying behaviour in freestall barn. J Dairy Sci 85, 2407-2413. 
Petersen G, D Nelson. 1984. Foot diseases in cattle. Part II. Diagnosis and treatment. The Compendium of Continuing Education. Food Animal Practice 6, 565-573.

Pyman M F S. 1997. Comparison of bandaging and elevation of the claw for the treatment of foot lameness in dairy cows. Aust Vet $J$ $75,132-135$

Radostits O M, C C Gay, D C Blood, K W Hinchcliff. 2000. Veterinary Medicine. A textbook of the diseases of Cattle, Sheep, Pigs, Goats and Horses. $9^{\text {th }}$ ed. WB Saunders, London, UK, Pp 948-958,

Rajala-Schultz P J, Y T Gröhn, C E McCulloch. 1999. Effects of milk fever, ketosis, and lameness on milk yield in dairy cows. J Dairy Sci 82, 288-294.

Rodríguez A, P Meléndez, D Hird, D Read, R Walter. 1999. Farm and host level risk factors for papillomatous digital dermatitis in Chilean dairy cattle. Prev Vet Med 42, 87-97.

Schultz T A. 1984. Weather and shade effects on cow corral activities. J Dairy Sci 67, 868-873.

Shearer J K. 1998. Lameness in dairy cattle: consequences and causes. The Bovine Practitioner 32, 79-84.

Sogstad A M, T Fjedaas, O Osteras. 2005. Lameness and claw lesions of the Norwegian red dairy cattle housed in free stalls in relation to environment, parity and stage of lactation. Acta Vet Scand 46, 203-217.

Sprecher D J, D E Hostetler, J B Kaneene. 1997. A lameness scoring system that uses posture and gait to predict dairy cattle reproductive performance. Theriogenology 47, 1179-1187.

Sumner J, R C Davies. 1984. Footbaths on dairy farms in England and Wales. Vet Rec 114, 88.

Tadich N. 2003. Claudicaciones en la vaca lechera y su relación con el bienestar animal. VI Jornadas Chilenas de Buiatría. Pucón, Chile.
Tadich N, E Hettich, G van Schaik. 2005. Prevalencia de cojeras en vacas de 50 rebaños lecheros del sur de Chile. Arch Med Vet 37, 29-36.

Tranter W P, R S Morris. 1991. A case study of lameness in three dairy herds. New Zeal Vet J 39, 88-96.

UACH, Universidad Austral de Chile 1999. Caracterización del rebaño lechero nacional. En: Competitividad de la producción lechera nacional. $\mathrm{Pp}$ 17-22.

Van Amstel S R, J K Shearer. 2001. Abnormalities of hoof growth and development. The Veterinary Clinics of North America. Food Animal Practice 17, 73-91.

Vermunt J J. 1990. Lesions and structural characteristics of the claws of dairy heifers in two management systems. MSc Thesis, University of Saskatchewan, Canada.

Vermunt J J. 1992. Subclinical laminitis in dairy cattle. New Zeal Vet $J$ 40, 133-138.

Vermunt J J, P R Greenough. 1994. Predisposing factors of laminitis in cattle. Brit Vet $J 150,151-164$.

Vermunt J J. 1999. Regular claw trimming for the control of lameness: good or bad. Vet $J 157,109-110$

Vermunt J J, T J Parkinson. 2002. Claw lameness in dairy cattle: New Zealand based research. New Zeal Vet J 50, suppl 88-89.

Warnick L D, D Janssen, C L Guard, Y T Gröhn. 2001. The effect of lameness on milk production in dairy cows. J Dairy Sci 84, 19881997.

Westwood C, E Bramley, I Lean. 2003. Review of the relationship between nutrition and lameness in pasture-fed dairy cattle. New Zeal Vet J 51, 208-218.

Whay H, D Main, L Green, J Webster. 2003. Assesment of the welfare of dairy cattle using animal-based measurement: direct observations and investigation of farm records. Vet Rec 153, 197-202. 
